\title{
Hidrodinámica aplicada a la gestión y la conservación de ecosistemas marinos y costeros: Isla Gorgona, Océano Pacífico Colombiano
}

Andrés F. Osorio A. ${ }^{*}$, Daniel Santiago Peláez-Zapata ${ }^{1}$, Juan Guerrero-Gallego ${ }^{1}$, Oscar ÁlvarezSilva $^{1}$, Juan David Osorio-Cano ${ }^{1}$, Francisco Mauricio Toro ${ }^{1} \&$ Alan Giraldo $^{2}$

1. Departamento de Geociencias y Medio Ambiente. Facultad de Minas. Universidad Nacional de Colombia sede Medellín; afosorioar@unal.edu.co,dspelaez@unal.edu.co,jjguerre@unal.edu.co,oaalvare@unal.edu.co, jdosori0@unal.edu.co,fmtoro@unal.edu.co.

2. Departamento de Biología, Universidad del Valle. Calle 13 \# 100-00, Cali. Colombia; oceanografia@univalle.edu.co, alan.giraldo@correounivalle.edu.co

Recibido 18-X-2013. Corregido 20-XI-2013. Aceptado 19-XII-2013.

\begin{abstract}
Hydrodynamics applied to the management and conservation of marine and coastal ecosystems: Gorgona Island, Colombian Pacific Ocean. Playa Palmeras (Gorgona National Park) is one of the most important sites for sea turtle nesting in South and Central America. Because of the morphological processes affecting the beach, the turtle nests are increasingly exposed to the impact of waves and tides, threatening conservation. A study was made to determine the hydrodynamical processes of the zone adjacent to Playa Palmeras, which affects directly the morphodynamical behavior of the beach and thus the preservation of the nests. Field measurements and numerical modeling were used to obtain the general circulation patterns and thermohaline structure behavior in the area in order to determine the spatial and temporal variability of waves and its effects on the beach. A marked seasonality was found, both in the waves and the currents, influenced mainly by the meridional displacement of the ITCZ (Inter-Tropical Convergence Zone) and an interannual variability of the waves, associated with ENSO (El Niño Southern Oscillation). The flooding levels of the beach were determined and flooding probability maps were made, where safer sites to locate the turtle nests could be identified. These maps serve the officials responsible of monitoring the turtles as a tool to take decisions on moving the nests, since they have flood risk information for any point on the beach. The results show that the middle zone north of the beach is the one with the lowest risk of flooding, therefore the most appropriate zone to relocate nests that are in high risk areas. Rev. Biol. Trop. 62 (Suppl. 1): 133-147. Epub 2014 February 01.
\end{abstract}

Key words: numerical modeling, circulation patterns, thermohaline structure, wave propagation, flooding level, coastal management.

El Parque Nacional Natural Gorgona (PNNG) es uno de los sitios más importantes para la anidación de tortugas marinas en América (Rueda-Almonacid et al., 2007). En especial Playa Palmeras, localizada en el costado suroeste de la isla (Fig. 1), es el lugar del Parque donde se registra el mayor anidamiento. En esta playa de $1.2 \mathrm{~km}$ de longitud se está presentando un sostenido proceso de erosión, de tal forma que los nidos de las tortugas se ven cada vez más expuestos al impacto del oleaje y la marea. Las tortugas marinas dependen de zonas de playa seguras para su correcta reproducción. Actualmente todos los nidos identificados son trasladados a los puntos más elevados de la playa, modificando la ubicación natural escogida por las tortugas para el desove y poniendo en riesgo su conservación. (Amorocho \& Merizalde, 2004).

La Universidad Nacional de Colombia y el CIMAD, con el apoyo de Parques Nacionales Naturales de Colombia, abordaron este problema de erosión en Playa Palmeras desde múltiples frentes que incluyeron el estudio 


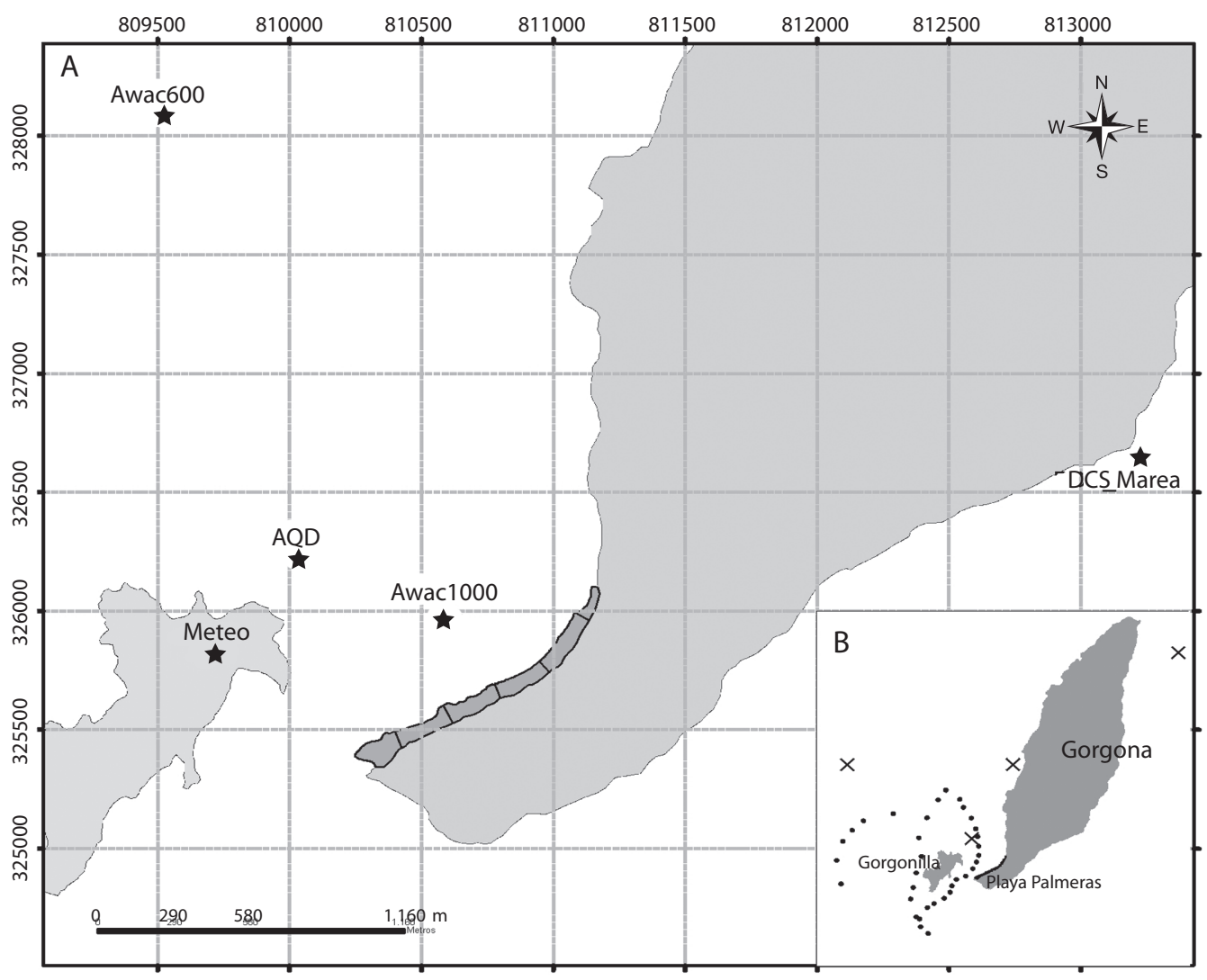

Fig. 1. Ubicación de la zona de estudio y de los equipos de medición de variables oceanográficas y climáticas. Sistema de coordenadas UTM Zona 17N. a). Ubicación de los equipos usados en las dos campañas de campo realizadas en el año 2011 (estrellas). b). Ubicación de los puntos donde se tienen perfiles de temperatura y salinidad tanto para la primera época del año (cruces) como para la segunda época del año (puntos).

Fig. 1. Location of the study area and equipment used to measure oceanographic variables. Coordinate system UTM $17 \mathrm{~N}$ Zone. a). Location of the equipments used in both field campaigns on 2011 (stars) b). Location of the measuring points of temperature and salinity profiles, for the first season of 2011 (crosses) and for the second season of 2011 (points).

de la hidrodinámica de oleaje y corrientes, el transporte de sedimentos y la evolución morfológica, la evolución de la vegetación de playa y el monitoreo de la anidación de las tortugas, todo esto con vistas al desarrollo de un plan de manejo integrado de la playa.

Este artículo se centra en el estudio de la hidrodinámica de las zonas marítimas circundantes al PNNG, especialmente en la zona cercana a Playa Palmeras. Se describe la climatología del oleaje en aguas profundas y los patrones de circulación asociados a la marea y los forzadores climáticos. Se estudiaron también los niveles de inundación de la playa asociados a distintas probabilidades de excedencia, generando mapas de los lugares seguros para la anidación de las tortugas, teniendo en cuenta la topografía de la playa. Otra aplicación del estudio de la hidrodinámica es estudiar el transporte de sedimentos y la evolución morfológica de la playa; estos últimos temas se describen brevemente en este artículo y se desarrollan en detalle en (Bernal et al., 2013 en prep.). Adicionalmente, Osorio et al., 2013 (en prep.), describen los resultados generales de todas las componentes de la 
investigación y el Plan Integrado de Manejo de la Playa elaborado.

\section{MATERIALES Y MÉTODOS}

Área de estudio: El PNNG se encuentra ubicado al sur del Océano Pacífico Colombiano a $36 \mathrm{~km}$ del punto más cercano del continente (Fig. 1). Tiene una extensión de $26 \mathrm{~km}^{2}$ de superficie terrestre conformada por la Isla Gorgona y el Islote Gorgonilla y $617 \mathrm{~km}^{2}$ de área marina. La climatología de Gorgona a escala intra-anual se ve influenciada fundamentalmente por la migración latitudinal de la Zona de Convergencia Intertropical (ZCIT), que en lugares extremos de la oscilación (como Gorgona), presenta un ciclo anual unimodal (Poveda, 2004; Blanco, 2009).

En los meses donde la ZCIT se encuentra en su posición más austral (diciembre-febrero), los vientos alisios provenientes del sureste son débiles en comparación a los vientos alisios del noreste. Al entrar estos vientos alisios en el Istmo de Panamá, se genera el chorro de viento de Panamá, que entra hasta el Pacífico Oriental Ecuatorial y domina el campo de vientos durante esta época (Amador, Alfaro, Lizano \& Magaña, 2006). En el caso de la época de junio a agosto, cuando la ZCIT está al norte, los vientos del sureste son los dominantes. Estos vientos cerca a los $3^{\circ} \mathrm{N}$ de latitud sufren un cambio de dirección, pasando a ser vientos del suroeste los cuales, en el occidente Colombiano, conforman un chorro de viento superficial, el Chorro del Chocó, el cual es el forzador dominante para el campo de vientos para esta época (Poveda \& Mesa, 1999; Amador et al., 2006).

Mediciones en campo: Se realizaron diferentes campañas de campo distribuidas a lo largo de 15 meses. Entre el 7 y el 11 de octubre de 2010 se realizó una campaña de diagnóstico en la cual fue posible la medición de algunas variables hidrodinámicas tales como oleaje y velocidades de flujo a partir de un correntómetro puntual Aquadopp $2.0 \mathrm{MHz}$ de la marca Nortek (AQD). Los cambios de marea se registraron a partir de un sensor de presión (DCX-marea, Fig. 1) ubicado en el sector de la azufrada y el cual permaneció a lo largo de todo el proyecto con el fin de lograr una serie continua para la validación y calibración de los modelos numéricos. De igual manera se midieron diferentes variables climáticas en intervalos de 10 min de radiación solar, temperatura del aire, humedad relativa, precipitación, presión atmosférica y velocidad y dirección del viento usando una estación meteorológica (Davis Vantage Pro) instalada en el faro de Gorgonilla (Fig. 1).

Con el fin de caracterizar tanto las variables locales que influencian los patrones de circulación y la estructura termohalina, como los cambios en los vientos y su efecto sobre el oleaje asociados al paso de la ZCIT, se realizaron dos campañas de campo adicionales durante el año 2011. La primera entre el 16 y el 24 de febrero para caracterizar la primera época del año (diciembre a marzo) y una segunda campaña entre el 12 y 22 de agosto para caracterizar la segunda época del año (abril a noviembre). Las variables oceanográficas medidas en campo fueron el oleaje (altura de ola, período y dirección), la variación del nivel del mar, perfiles verticales de corrientes y variación de la temperatura y la salinidad en varios puntos en la zona de estudio (Fig. 1).

Para las mediciones de oleaje, marea y corrientes se usaron tres sensores acústicos de la marca Nortek: un AWAC $1.0 \mathrm{MHz}$ (Awac1000), un AWAC 600kHz (Awac600) y un correntómetro puntual Aquadopp $2.0 \mathrm{MHz}$ (AQD). El oleaje se midió en estados de mar de una hora a partir de registros durante 1024s (17min aprox.), mientras que las corrientes se registraron cada $30 \mathrm{~min}$ promediando durante 120 s. Los perfiles de salinidad y temperatura fueron medidos para la segunda época del año mediante una sonda multiparamétrica HYDROLAB MS5. Para el caso de la primera época del año, en la cual no fue posible la medición de perfiles de salinidad y temperatura, se usaron las mediciones de estas variables realizadas por el grupo de investigación en Ciencias Oceanográficas de la Universidad 
del Valle durante el monitoreo oceanográfico semestral, entre el 4 y el 6 de marzo de 2011, debido a que en un lapso de menos de un mes (entre el período de simulación y las mediciones) no se esperan cambios significativos en la temperatura y la salinidad. En la Fig. 1 se presenta la ubicación de los equipos de medición empleados y los puntos de muestreo para los perfiles de salinidad y temperatura.

Además de la información hidrodinámica recopilada en campo, se realizó un levantamiento topo-batimétrico a partir de un GPS con corrección diferencial (TopconHiperLite+), entre la línea de baja marea y la zona de vegetación. Por otra parte, se complementó la información existente de las cartas náuticas, proporcionadas por la DIMAR (Dirección General Marítima), del Ministerio de Defensa Nacional de Colombia, con nuevos puntos batimétricos entre isla Gorgona y Gorgonilla, empleando una ecosonda monohaz marca Hummingbird. Dicha batimetría fue corregida a partir de la serie de marea medida en campo y referenciada al promedio de las Bajamares de sicigia (MLLW) según la serie histórica de marea en Tumaco (1990-2008), que es el sitio más cercano con registro de marea de largo plazo disponible en la zona.

Hidrodinámica de corrientes: Se estudió a partir de modelación hidrodinámica tridimensional de un mes característico de cada una de las dos épocas del año. La información necesaria para la modelación (nivel de marea, perfiles de salinidad, temperatura y corrientes) se obtuvo principalmente de mediciones realizadas en las campañas de campo (descritas en la sección anterior), aunque fue necesario usar información secundaria, proveniente de bases de datos y de trabajos realizados por otros autores en la zona para fechas coherentes con los escenarios planteados. Las variables meteorológicas que no fue posible medir (índice de nubosidad para ambas épocas del año y la radiación solar para la segunda época del año), se obtuvieron del proyecto de reanálisis climático NCEP/ NCAR (Kalnay et al., 1996). Las corrientes geostróficas se obtuvieron de mediciones de altimetría satelital realizadas por Ssalto/Duacs y distribuidos por Aviso, con el apoyo del Centre National d'Etudes Spatiales (Cnes; http://www.aviso.oceanobs.com/duacs/). Estas corrientes se usaron como condiciones iniciales y de frontera para el modelo, dado que estas sirven como una aproximación para las corrientes oceánicas que pueden ser observadas en esta zona. Para la batimetría se usaron datos de las cartas náuticas proporcionadas por la DIMAR, los cuales fueron interpolados al dominio de cálculo definido para este caso.

Para la modelación hidrodinámica se utilizó el modelo ELCOM (Estuary, Lake COastal Model) desarrollado por el Centre of Water Research (CWR) de la University of Western Australia. Este modelo hidrodinámico resuelve las ecuaciones tridimensionales de NavierStokes para un flujo no permanente, viscoso e incompresible, usando la aproximación hidrostática para la presión y la técnica de descomposición de Reynolds. Para la solución numérica de las ecuaciones usa el esquema semi-implícito de diferencias finitas desarrollado por Casulli \& Cheng (1992). Este modelo simula los procesos hidrodinámicos y termodinámicos que se desarrollan en cuerpos de agua estratificados, sometidos a la acción de forzadores físicos, dando una representación del comportamiento espacial y temporal de variables como la velocidad, la temperatura y la salinidad en estos cuerpos de agua (Hodges \& Dallimore, 2006).

Se hicieron análisis de sensibilidad a los parámetros físicos y numéricos del modelo para garantizar que los resultados describan de forma adecuada los procesos hidrodinámicos y termodinámicos que se desarrollan en la zona. A partir de estos análisis de determinó el tamaño del dominio de cálculo, el tamaño de las celdas de cálculo en sentido vertical y horizontal y el paso de tiempo adecuado para que los resultados obtenidos sean numéricamente estables y físicamente representativos. El dominio de cálculo se definió como una región de $20 \mathrm{~km}$ x $20 \mathrm{~km}$, centrado en el estrecho de Tasca, con tamaño de celda variable en la horizontal desde $500 \mathrm{~m}$ de lado en las fronteras 
hasta $100 \mathrm{~m}$ en la zona de detalle. Para el caso de la discretización vertical se trabajó también con un tamaño variable, tratando de hacer una mejor representación de las aguas superficiales, donde hay mayor variabilidad, teniendo celdas de tamaño de $0,75 \mathrm{~m}-1,2 \mathrm{~m}$ en superficie. Para el caso del paso de tiempo se pudieron obtener resultados estables con $45 \mathrm{~s}$.

Una limitación importante de este modelo es que las condiciones de frontera de mareas y de corrientes no son compatibles, por lo que solo puede haber una de estas condiciones en cada frontera. Por esta razón, se evaluaron las condiciones de corrientes y con base en las direcciones predominantes en cada época, se determinaron las fronteras forzadas por corrientes y aquellas forzadas por marea. Las fronteras transversales a la componente predominante de cada época fueron configuradas como las fronteras con la condición de corrientes (una frontera para la entrada de la corriente y otra para la salida). Las fronteras restantes se configuran con la condición de nivel impuesta por la marea.

El modelo ELCOM se calibró utilizando las mediciones de los forzadores físicos y las características del agua registradas entre el 1 y 23 de febrero de 2011 y se validó con las mediciones realizadas entre el 1 y 22 de agosto de 2011. De esta forma se busca garantizar la representación adecuada de los procesos hidrodinámicos que se desarrollan en las diferentes épocas del año. En la Fig. 2 se muestra la comparación de un caso representativo de un perfil de salinidad tanto para la primera época (período de calibración del modelo), como para la segunda época del año (período de validación). En esta figura se pueden observar diferencias notorias en el comportamiento de las características oceanográficas de la zona, las cuales se entrelazan con el comportamiento que exhiben las variables meteorológicas.

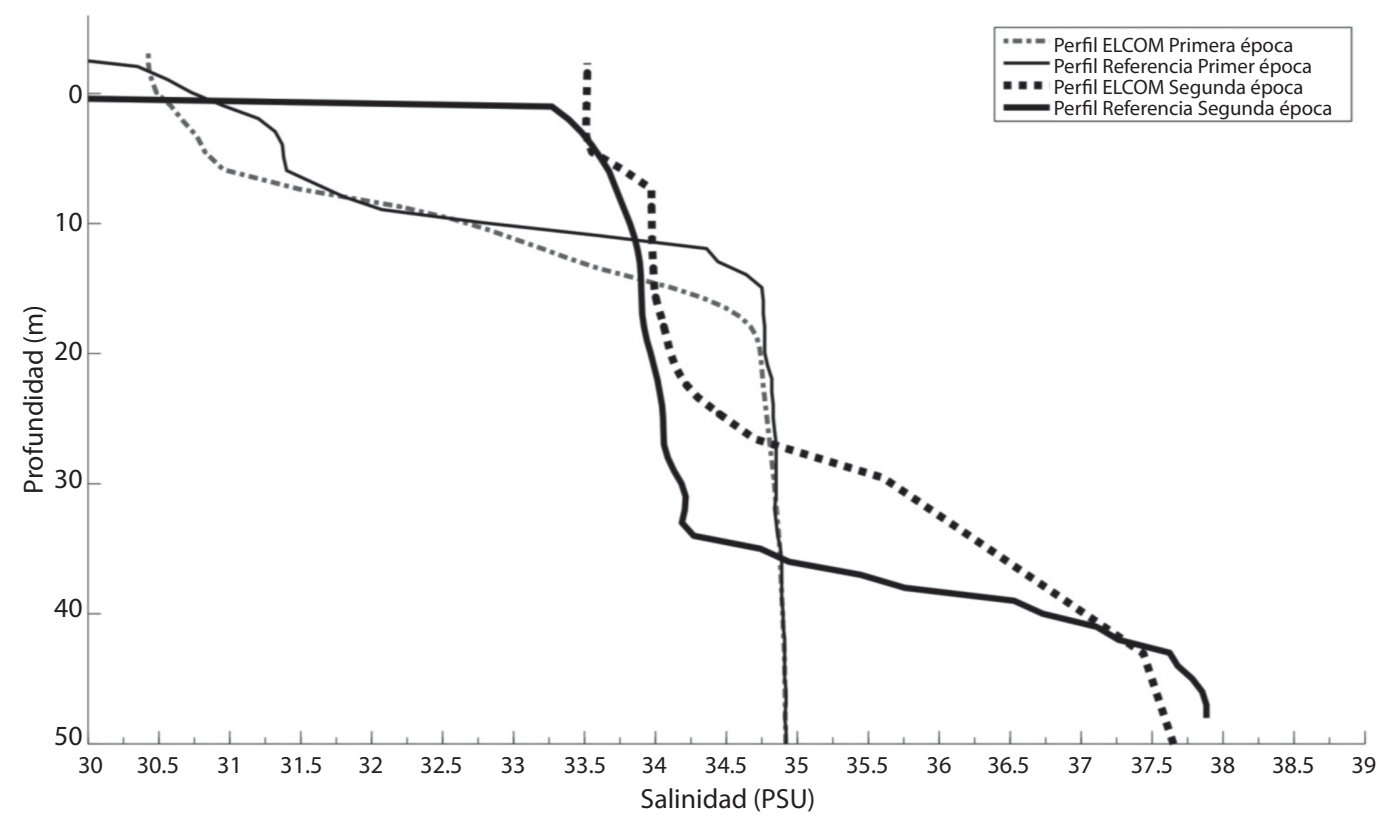

Fig. 2. Comparación de un caso representativo de perfiles de salinidad, medido vs. modelado. a). Período de calibración (Primera época) b). Período de validación (Segunda época).

Fig. 2. Comparison of a representative case of salinity profiles, measured vs. simulated: a). Calibration period (First season) b). Validation period (Second season). 
Hidrodinámica del oleaje: Para determinar la variabilidad intra-anual e interanual del oleaje en aguas profundas, se usó una serie de altura de ola significante (Hs), período pico (Tp) y dirección (Dir) de la base de datos de reanálisis global del proyecto GOW (Globe Ocean Waves) del IH Cantabria, en el punto $78,5^{\circ} \mathrm{W}-3,0^{\circ} \mathrm{N}$ cerca del PNN Isla Gorgona (Reguero, Menéndez, Méndez, Mínguez \& Losada, 2012). La serie tiene una resolución horaria, con datos desde 1948 hasta 2008, validados con boyas de la NDBC (National Data Buoy Center) de la NOAA. De esta serie se obtuvo el ciclo medio mensual multianual del oleaje (Hs, Tp, Dir) y su variabilidad interanual asociada al ENSO. Se usó el Índice Multivariado del ENSO (MEI; Multivariate ENSO Index (Wolter \& Timlin, 1998) para determinar los años El Niño, La Niña y Normales.

Para conocer la dinámica del oleaje en Playa Palmeras, se usó el modelo SWAN (Simulating WAves Nearshore; Booij, Ris \& Holthuijsen, 1999) para propagar 21 años (1980-2000) de oleaje desde aguas profundas $\left(78,5^{\circ} \mathrm{W}-3,0^{\circ} \mathrm{N}\right)$ hasta pie de playa. Éste modelo está diseñado para simular la propagación del oleaje cerca de la costa y reproduce de manera adecuada los procesos de generación y transformación del oleaje (The SWAN Team, 2009). El modelo fue forzado con vientos a 10msnm del reanálisis NCEP/NCAR (Kalnay et al., 1996). Para la batimetría se usaron datos del modelo mundial de relieve ETOPO1 (Amante \& Eakins, 2009) combinados con levantamientos de detalle realizados en campo y con cartas náuticas levantadas por la DIMAR

Se usó un esquema de cuatro mallas anidadas, pasando de resolución de $700 \mathrm{~m}$ hasta $50 \mathrm{~m}$. Se utilizaron condiciones de frontera paramétricas para la primera malla (oleaje en aguas profundas) y espectral en las siguientes (resultados de las mallas precedentes). La ejecución del modelo se hizo en modo no estacionario, considerando los procesos de refracción, difracción, white-capping, disipación por fondo y por rotura e interacción no lineal entre olas, con parámetros definidos por defecto. Los resultados del modelo fueron validados en el dominio de la probabilidad con las mediciones de oleaje realizadas en febrero de 2011. Se comparó la distribución estadística de Hs medida y los resultados de la simulación de los meses de febrero de cada año, utilizando los cuantiles del $1 \%$ de cada serie.

Probabilidad de inundación: Se usó una serie horaria de un mareógrafo ubicado en el municipio de Tumaco en la costa Pacífica colombiana cerca a Gorgona, con datos de nivel del mar desde 1951 hasta 2000, con un $15 \%$ de datos faltantes. Estos datos se validaron con mediciones en campo (AQD) y se encontró que la marea en Gorgona es aproximadamente la misma que en Tumaco. La marea se descompuso en sus armónicos principales usando series de Fourier, para determinar las componentes astronómica y meteorológica. Se comparó la marea meteorológica a escala interanual con el MEI.

La cota de inundación se calculó como la suma del nivel de marea (astronómica y meteorológica) y la altura hasta donde asciende el oleaje al llegar a la playa (run-up). Se utilizó la serie de oleaje propagada hasta pie de playa (1980-2000) y la serie de marea registrada por el mareógrafo de Tumaco para el mismo período de tiempo, tomando como nivel de referencia el promedio de bajamares de "sicigia" del registro de marea. El run-up se calculó usando una formulación empírica (Nielsen \& Hanslow, 1991), la cual depende de Hs, Tp y la pendiente media de la playa $(\tan \beta)$. Se usó la pendiente media de la playa $(\tan \beta=0.12)$ obtenida del levantamiento topográfico detallado.

\section{RESULTADOS}

Hidrodinámica de Corrientes: En la Fig. 3a se observa el patrón medio de circulación en la primera época para la zona de detalle (énfasis a la zona del estrecho de Tasca y Playa Palmeras), destacándose que la dirección de las corrientes resultantes de la superposición de efectos de las variables meteorológicas, las mareas, las corrientes oceánicas y los procesos termohalinos es predominante hacia el Este. Adicionalmente, se identificó un marcado 
A

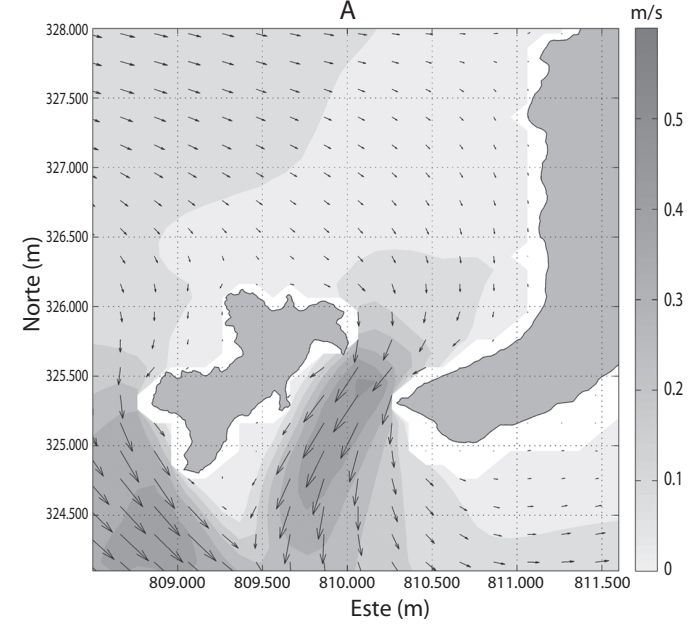

B

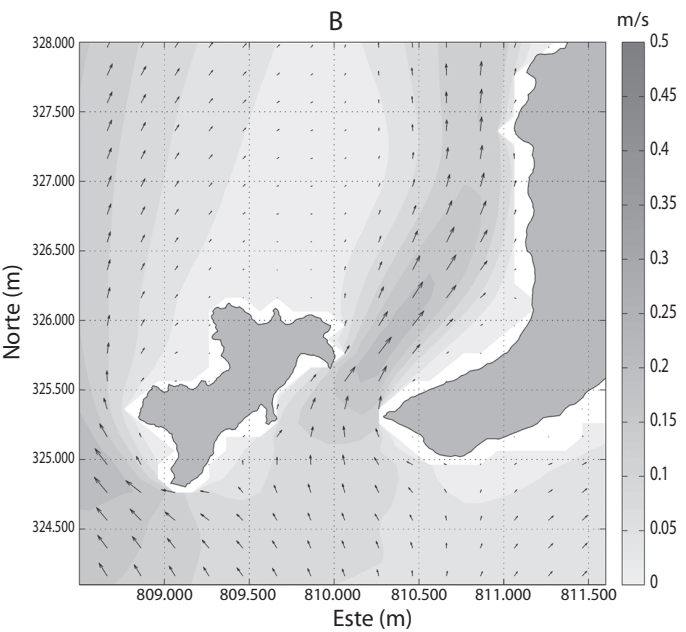

Fig. 3. Patrón de circulación promedio en Isla Gorgona, en la zona de detalle del estrecho de Tasca y la Playa Palmeras, para ambas épocas del año 2011. Sistema de coordenadas: UTM, zona 17N. La escala de grises representa la magnitud de la velocidad de las corrientes: a). Patrón promedio de corrientes para el mes de febrero de 2011, que representa la primera época del año; b). Patrón promedio de corrientes para el mes de agosto de 2011, que representa la segunda época del año. Fig. 3. Mean circulation pattern in Gorgona Island, a detail of interest zone of the Tasca Strait and Palmeras Beach, for the two seasons of 2011. Coordinates System: UTM, Zone 17N. The gray scale represents the magnitude of the current velocity: a). Mean current pattern on February 2011, which represents the first season of the year. b) Mean current pattern on August 2011, which represents the second season of the year.

efecto de la batimetría sobre el patrón general de circulación, generando cambios en la dirección de las corrientes en aguas someras próximas a Isla Gorgona y el islote Gorgonilla (Fig. 3a). En términos generales, las corrientes superficiales se dirigen hacia isla Gorgona, con dirección suroeste en el estrecho de Tasca, recostándose en la parte sur de Playa Palmeras y en el oeste sobre Playa Gorgonilla (Fig. 3a). El islote de Gorgonilla actúa como punto de refracción y de difracción de las corrientes, dado que divide las corrientes y les cambia la dirección (Fig. 3a). En este sector, la corriente tiende a fluir hacia el sur por el estrecho de Tasca, con una mayor magnitud hacia el centro del estrecho. Además, la marea tiene en este caso un papel modulador de las magnitudes de las corrientes, afectando principalmente el foco de velocidades mayores en el centro del estrecho de Tasca, el cual cambia a medida que se propaga la onda de marea alrededor de isla Gorgona.

Los patrones de circulación en la segunda época del año (Fig. 3b), muestran que las corrientes resultantes, (de nuevo haciendo énfasis en la zona del estrecho de Tasca y Playa Palmeras), por la superposición de efectos de las variables meteorológicas, las mareas, las corrientes oceánicas y los procesos termohalinos tiene dirección predominante hacia el Norte. Durante este periodo también es notorio el efecto de la batimetría sobre el patrón general de circulación, generando cambio de dirección de las corrientes en aguas muy someras (Fig. 3b). Al revisar todos los resultados de la simulación se nota que en general la tendencia de la corriente es a fluir hacia el Norte por el estrecho de Tasca, oscilando del Noreste (hacia la parte Norte de Playa Palmeras) y en algunos días hacia el Noroeste (hacia Gorgonilla) (Fig. $3 b)$. En este periodo también las corrientes se recuestan en el Este sobre Playa Palmeras y en el Oeste sobre Playa Gorgonilla. La mayor magnitud de las corrientes es hacia el centro del estrecho de Tasca, aunque en algunos periodos se mueve el área de mayor magnitud hacia afuera del estrecho de Tasca, específicamente hacia el Sur de isla Gorgona. El efecto de 
marea, de manera similar que durante el primer periodo del año, concentran el foco de máximas velocidades en el centro del estrecho de Tasca.

Hidrodinámica de oleaje: El clima marítimo en aguas profundas en Isla Gorgona (Fig. 4) tiene un marcado ciclo anual determinado por la migración latitudinal de la ZCIT. En el trimestre diciembre-enero-febrero (DEF) el recurvamiento de los vientos alisios del noreste hacia el Este genera que parte del oleaje en esta época provenga del Norte, con alturas de ola en promedio de $0.6 \mathrm{~m}$ y períodos de $12 \mathrm{~s}$. En el trimestre Mar-Abr-May (MAM) se presentaron las menores Hs (cerca de $0.5 \mathrm{~m}$ ) y los mayores Tp (hasta 13s), se presentó oleaje predominante del SW, producto del recurvamiento de los vientos alisios del sureste. A partir del trimestre Jun-Jul-Ago (JJA), la altura de ola empezó a crecer debido a que se intensifican los vientos del chorro del Chocó, llegando a un máximo en el trimestre Sep-Oct-Nov (SON), donde el oleaje significante alcanzó en promedio $0.75 \mathrm{~m}$ de altura y períodos de $10 \mathrm{~s}$. En general, en los trimestres DEF y MAM se presenta un oleaje con menor influencia de los vientos de la zona, predominando el oleaje de fondo, mientras que en los trimestres JJA y SON se presenta una influencia directa del chorro del Chocó. Existe también una marcada variabilidad del ciclo anual del oleaje asociada al ENSO; se observa que, en general, el oleaje es más débil durante la fase cálida del ENSO y más fuerte durante la fase fría. Esto se debe a que los vientos alisios se debilitan durante los años El Niño y se intensifican en los años La
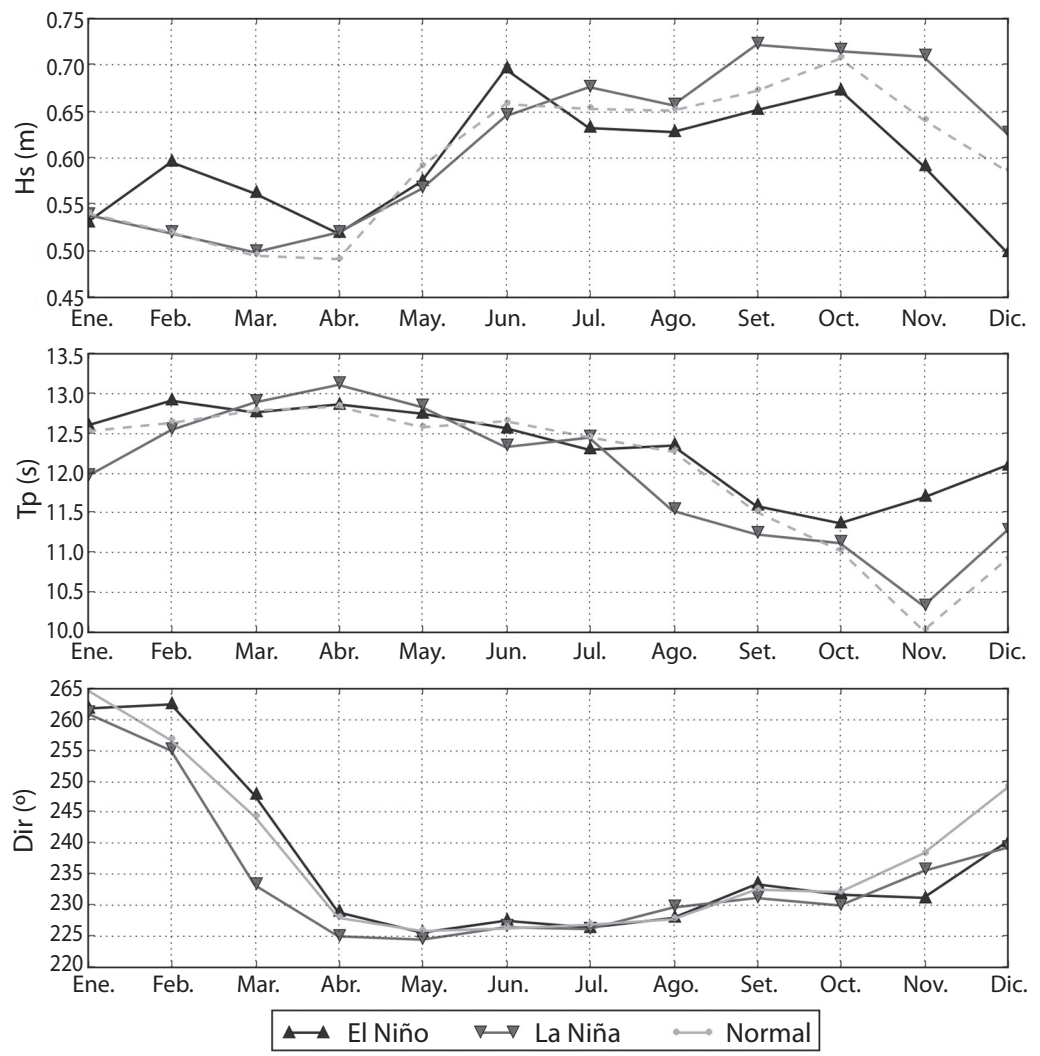

Fig. 4. Ciclo anual de altura de ola significante (Hs), período pico (Tp) y dirección (Dir) en Isla Gorgona y su variabilidad interanual asociada a las fases cálida y fría del ENSO.

Fig. 4. Annual cycle of significant wave height (Hs), peak period (Tp) and direction (Dir) in Gorgona Island and its interannual variability caused by the warm and cold phases of the ENSO. 
Niña (Poveda \& Mesa 1999, Poveda 2004), generando estas diferencias en la altura de ola entre las dos fases. En los meses de febrero, marzo y junio durante los años El Niño se presentan las mayores alturas de ola, contrario a los que sucede en los otros meses.

Los resultados de la validación de la propagación de oleaje desde aguas profundas hasta Playa Palmera se presenta en la Fig. 5. Se observó que la simulación subestimó los máximos y sobrestimó los mínimos, pero en general ambas distribuciones fueron similares entre sí. En la Fig. 5C se representa el campo promedio de Hs y dirección (SWAN) en la malla más fina $(50 \mathrm{~m})$ para cada trimestre. En el trimestre DEF el oleaje promedio fue del W, ya que gran parte del oleaje llegó del norte; mientras que en el trimestre SON fue del SW y se presentó una mayor magnitud comparada con las otras épocas del año. Se estableció que el islote Gorgonilla funciona como protección para Playa
Palmeras, ya que la dirección predominante del oleaje fue del SW, y la presencia del islote generó una disminución de hasta $0.3 \mathrm{~m}$ en la altura de ola que alcanza Playa Palmeras. Se observó que las mayores Hs de la zona se presentaron antes de Gorgonilla, debido a bajos en la batimetría estarían generando una reducción importante de la profundidad.

Probabilidad de inundación: La cota de inundación estuvo controlada generalmente por el oleaje (run-up), aunque la marea meteorológica genera sobre-elevaciones significativas en el nivel del mar (Fig. 6A). En una escala interanual, la variabilidad de la cota de inundación estuvo influenciada por el ENSO, que afecta directamente la marea meteorológica. Los mayores niveles de inundación se presentaron en los años que corresponden a la fase cálida (El Niño) y los menores en la fase fría (La Niña). Se encontró una correlación simultánea
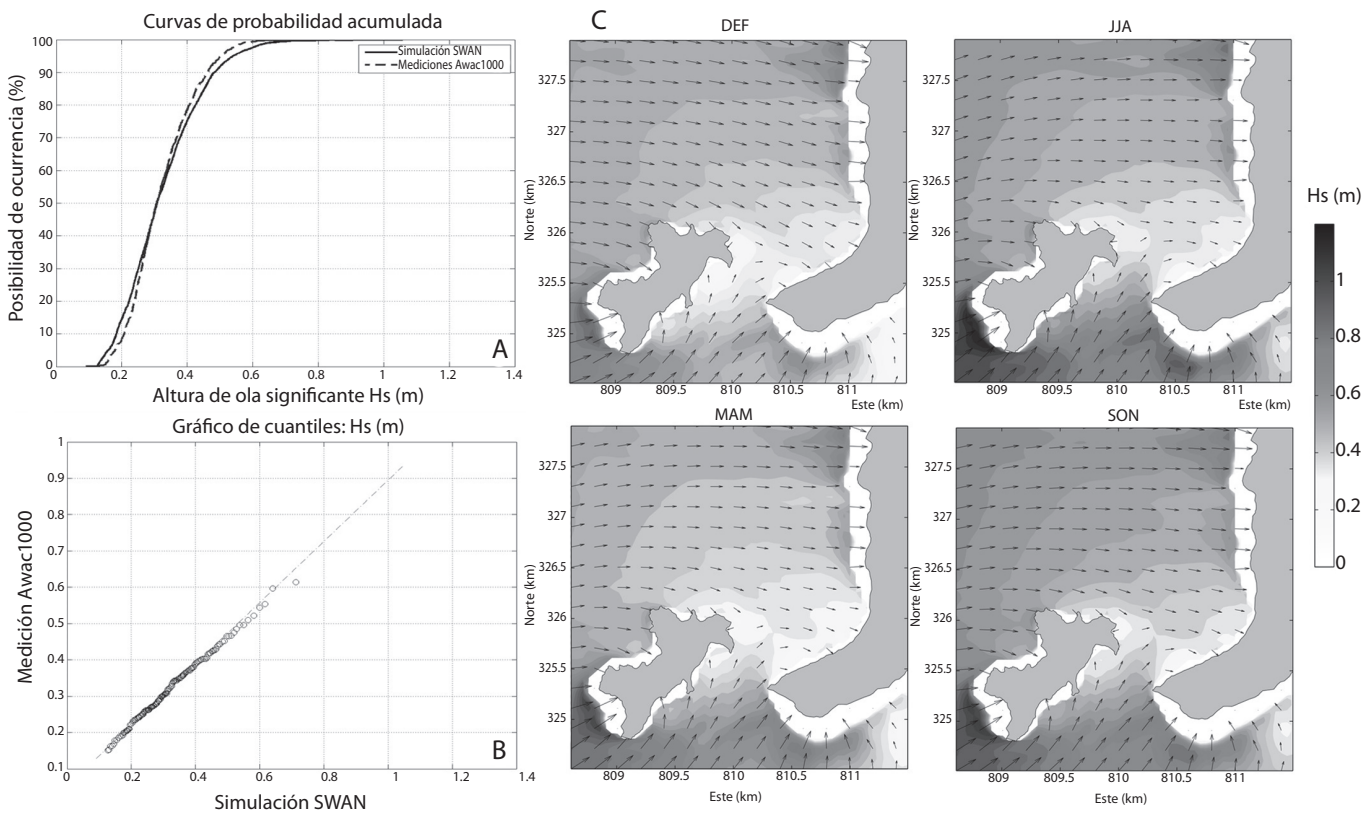

Fig. 5. Resultados de la propagación de oleaje desde aguas profundas hasta pie de playa. A) Curvas de distribución de probabilidad acumulada para la serie medida (Awac1000) y simulada (SWAN); B) Gráfico de cuantiles del 1\% para las dos series. C) Campo de altura de ola significante de la zona de estudio para cada trimestre. Sistema de coordenadas UTM, zona $17 \mathrm{~N}$.

Fig. 5. Results of wave propagation from deep water to nearshore. A) Curves of cumulative probability distribution of measured (Awac1000) and simulated (SWAN) time series; B) Quantile-Quantile plot associated with $1 \%$ of both distributions. C) Seasonal significant wave height field of the study area. Coordinate System UTM, Zone 17N. 

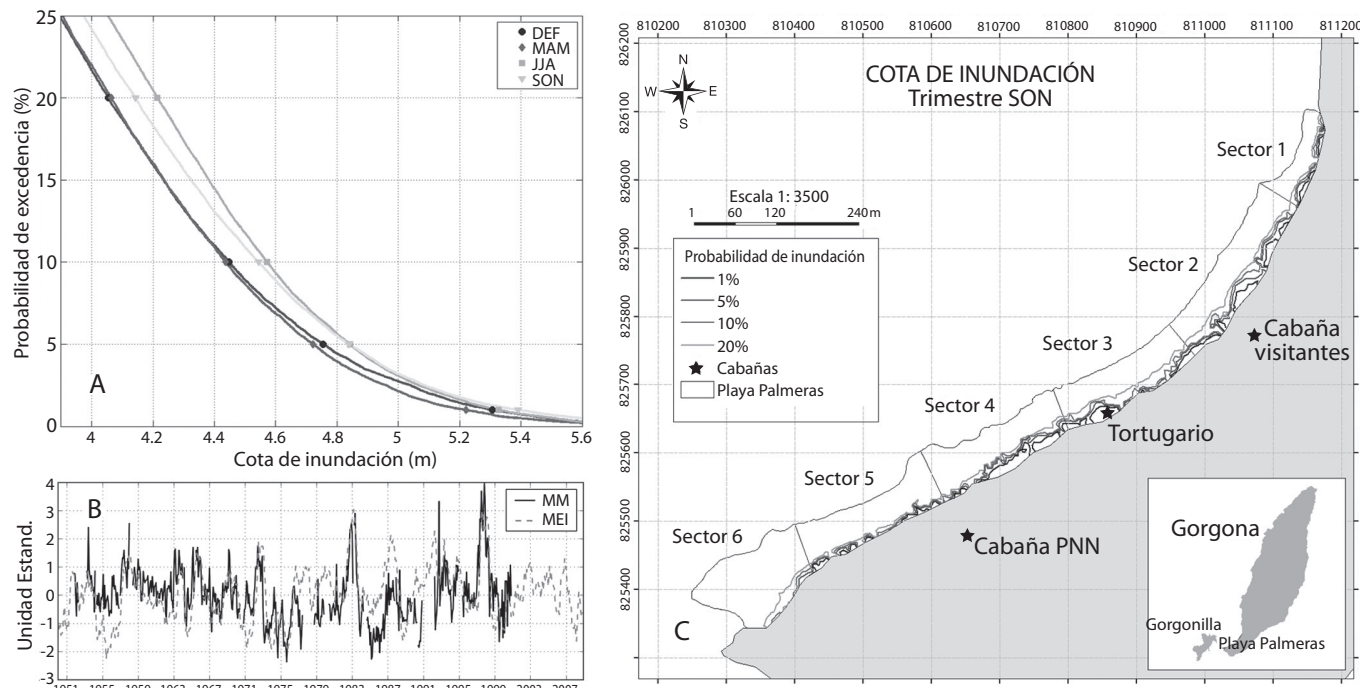

Fig. 6. Cota de inundación de la zona de estudio. A) Curvas de probabilidad de excedencia de la cota de inundación para cada trimestre; B) Correlación simultánea entre el Índice Multivariado del ENSO (MEI) y la marea meteorológica (MM); C) Mapa de cota de inundación de Playa Palmeras para 1\%, 5\%, 10\%, 20\% de probabilidad de excedencia para el trimestre SON. Sistema de coordenadas UTM, zona $17 \mathrm{~N}$.

Fig. 6. Flood elevation of the study area. A) Seasonal exceeding probability curves of the flood elevation; B) Simultaneous correlation between Multivariate ENSO Index and meteorological tide; C) Flood elevation map of Playa Palmeras for 1\%, $5 \%, 10 \%, 20 \%$ probability of exceedance for SON quarter. Coordinate System UTM, Zone $17 \mathrm{~N}$.

entre el MEI y la marea meteorológica del 51,8\% (Fig. 6B). El ENSO, además, presentó una variabilidad estacional ya que comienza en los meses de marzo-mayo y alcanza su máximo desarrollo durante los meses de noviembreenero (Poveda, 2004), esto se observa en la Fig. $6 \mathrm{~A}$, donde se muestra un aumento considerable en los trimestres SON y DEF para las cotas de inundación excedidas menos del 5\% del tiempo. Esto sugiere que los máximos de la cota de inundación son controlados por la marea meteorológica y a su vez por el ENSO.

Se construyeron mapas de probabilidad de inundación asociadas a probabilidades de excedencia del $1 \%, 5 \%, 10 \%$ y $20 \%$ en la topografía de la playa para cada trimestre. En el trimestre SON se identificaron las condiciones más críticas (Fig. 6C), estableciéndose los niveles más altos de cota de inundación, correspondiendo con el periodo en donde se registra la mayor cantidad de anidamientos de tortugas. Se identificó que las zonas con menor probabilidad de inundación se encuentran hacia el sector medio-norte de la playa, definiendo en estos sitios las áreas más seguras para el anidamiento natural de las tortugas y para el traslado de los nidos desde zonas de mayor riesgo (Fig. 6C). Por otro lado, en los sectores 5 y 6 , al sur de la playa las probabilidades de inundación son mucho mayores. Esta zona según las observaciones de campo, es donde se presenta mayor erosión y retroceso de la línea de vegetación.

Evolución morfológica de la playa: Las corrientes asociadas al oleaje, la marea y los demás forzadores ambientales son el motor del transporte de sedimentos en la playa y por tanto de su evolución morfológica (acreción y/o erosión de la playa). Los resultados de la hidrodinámica de Playa Palmeras descritos en este artículo se utilizaron para explicar el balance sedimentario de la Playa en el corto plazo y en el medio plazo, validando las corrientes y el transporte potencial asociado con mediciones periódicas de perfiles de playa; también se 
utilizaron para explicar las causas de la erosión de la playa, las tasas de erosión y el destino de los sedimentos que salen del sistema; y por último, se utilizaron para determinar las tendencias de la forma en planta de equilibrio de la playa en el largo plazo en comparación con la configuración actual (Bernal et al., 2013 en prep.).

\section{DISCUSIÓN}

Forzantes de mesoescala: Además de la influencia directa que tiene en el oleaje, la migración meridional ZCIT también afecta la configuración de la estructura termohalina de la columna de agua y ejerce un efecto modulador en el patrón de vientos de la zona a escala estacional, las cuales son condiciones que afectan el desarrollo de las corrientes superficiales locales (Wyrtki, 1966; Rodríguez-Rubio \& Stuardo, 2002; Amador et al., 2006; Chaigneau, Abarca del Rio \& Colas, 2006; Kessler, 2006). Los mayores rasgos observados en la variación de la profundidad de la termoclina en la zona, son resultado de una señal que se propaga en dirección suroeste generada por una onda Rossby provocada por el efecto combinado del incremento de la fuerza de los vientos a su paso por los cañones montañosos de Centroamerica, superpuesto por el forzamiento de la oscilación de la ZCIT, y que se manifiesta mediante el efecto que el viento tiene sobre la columna de agua, vía el bombeo de Ekman (surgencias en el caso de la zona de la Ensenada de Panamá) y la generación de dinámicas lineales de Sverdrup (Kessler, 2006).

A escalas estacionales se observa que la circulación en la zona está fuertemente controlada por la actividad de los vientos en Chorro que se desarrollan en la zona, el Chorro de Viento de Panamá y el Chorro del Chocó (Chaigneau et al., 2006; Devis-Morales, Schneider, MontoyaSánchez \& Rodríguez-Rubio, 2008). El primero genera un giro ciclónico y enfriamiento de las aguas en el centro de la Ensenada de Panamá a través de una fuerte evaporación y la entrada de aguas más frías desde la termoclina, mientras que el segundo, que está formado por vientos más fríos, interactúa con los vientos del noreste, que son más cálidos, lo que causa inestabilidad atmosférica considerable, generando grandes cantidades de precipitación en la región (Devis-Morales et al., 2008). Por estas condiciones de forzamiento físico, imperantes en la zona de la Ensenada de Panamá, se distinguen dos épocas oceanográficas opuestas en la zona de isla Gorgona, la primera entre enero y abril, caracterizada por aguas superficiales frías y con una alta salinidad y la segunda comprendida entre mayo y diciembre, caracterizada por aguas superficiales cálidas y de baja salinidad (Giraldo, Rodríguez-Rubio \& Zapata, 2008; Giraldo, Payán \& Chirimia, 2010).

Por otra parte, en algunas zonas costeras, el forzamiento físico de la marea es responsable de un alto porcentaje de la variabilidad de la superficie del mar, además de generar corrientes asociadas (Le Provost, 2001). En el caso particular del Pacífico Colombiano, la marea presenta un régimen meso-mareal semidiurno con carrera de marea media de $2.5 \mathrm{~m}$ en Tumaco y $3.5 \mathrm{~m}$ en Buenaventura, pasando al régimen macro-mareales durante las mareas vivas cuando se pueden alcanzar los $3.9 \mathrm{~m}$ y $4.9 \mathrm{~m}$ en Tumaco y Buenaventura respectivamente (Martínez, González, Pilkey \& Neal, 1995). Numerosos estudios han demostrado la gran importancia de este forzador en la hidrodinámica y morfodinámica de las zonas costeras y la plataforma continental somera en el litoral Pacífico Colombiano (Correa \& Gonzalez, 2000; Martínez \& López-Ramos, 2011; Restrepo \& Kettner, 2012).

La variabilidad climática a escala interanual en el Pacífico Colombiano está directamente relacionada con la ocurrencia del ENSO (El Niño-Oscilación del Sur). El ENSO afecta los patrones de circulación de vientos, lo cual se ve reflejado en la magnitud del oleaje. Algunos autores han estudiado el impacto del ENSO en las variables hidroclimáticas en el Pacífico Colombiano, entre ellos RodríguezRubio (2013) quien estudió la variabilidad climática a escalas interanual y decadal en una zona del Pacífico Colombiano y CorredorAcosta et al., (2011) quienes estudiaron las variaciones de las corrientes superficiales en la 
Ensenada de Panamá durante las fases cálida y fría del ENSO. El ENSO presenta oscilaciones de escala estacionales (por el ajuste de fase con el ciclo anual), cuasi-bienales (2 a 2,5 años), interanuales ( 2 a 8 años) y decadales (Poveda, 2004; Rodríguez-Rubio, 2013).

Hidrodinámica local y gestión para la conservación: El modelo ELCOM está diseñado especialmente para zonas estuarinas, lacustres y costeras (Hodges \& Dallimore, 2006), por lo que este estudio se puede considerar como una actividad de validación del modelo para escenarios de aguas oceánicas someras. Sin embargo a pesar de las restricciones para aplicaciones oceánicas, se eligió este modelo porque tiene un esquema numérico bien desarrollado, y formulaciones para la turbulencia y el transporte que representan de forma satisfactoria estas dinámicas, además que el modelo ya se ha usado en varias aplicaciones de carácter costero en el país (e.g. Montoya \& Toro, 2006; Gómez-Giraldo et al., 2009; Álvarez-Silva, Gómez-Giraldo \& Toro, 2010). A pesar de que la aproximación utilizada para la representación de las condiciones de frontera para la modelación no es exacta para las condiciones que existen en el océano, dada la configuración del dominio de cálculo y teniendo unas fronteras alejadas de la zona de interés, ésta se considera suficiente para la reproducción de los patrones de circulación medios en la zona de interés, donde pueden existir efectos locales debidos a la aproximación usada. Finalmente el efecto batimétrico contribuye también a dar forma a los patrones medios de circulación dentro del dominio.

Se puede considerar que el modelo ELCOM para los escenarios propuestos describió la física de la zona de manera razonable, ya que como se puede observar en la Fig. 2, describe en general los cambios que exhibe la estructura halina, mostrando una configuración de la estratificación que se aproxima a la que se puede observar en campo, la cual tiene una marcada variación intra-anual. De manera similar a la representación de la salinidad, el modelo es capaz de dar una representación de la estructura térmica de la columna de agua en la zona adyacente a Isla Gorgona. Al tener una representación razonable de la estructura termohalina, se puede considerar que la representación que se obtiene de las corrientes por medio del modelo es similarmente apropiada, debido a que la forma en que se desarrollan las corrientes depende de la densidad del agua, la cual para el caso del océano es función principalmente de la salinidad y en menor medida de la temperatura y la presión. Además, la escogencia de la salinidad y la temperatura como variables patrón es justificable desde el punto de vista de la búsqueda de la reproducción de los patrones de condiciones medias, es decir escalas temporales de menor detalle, donde se puede trabajar con variables más estables en lapsos cortos.

En general estas actividades de simulación con el modelo ELCOM permitieron resultados que dan una adecuada representación de la hidrodinámica de corrientes en la zona. Sin embargo, se requieren más simulaciones en otros sitios de la isla para validar el uso de este modelo en escalas temporales de más detalle. Adicionalmente, se logró un acercamiento adecuado para conocer las dinámicas asociadas a la circulación oceánica que afecta a isla Gorgona, elementos que pueden servir como punto de partida para más trabajos de modelación hidrodinámica en la zona, que permitan evaluar la variabilidad local de los patrones de circulación asociados a los sistemas ENSO y $\mathrm{PDO}$, o relacionados con otros sistemas como la Ensenada de Panamá, como los plantean Rodríguez-Rubio, Schneider \& Abarca del Rio (2003) y Devis-Morales et al. (2008), o incluso el efecto del sistema deltaico Patía-Sanquiaga como lo sugiere Giraldo et al. (2008) y Restrepo \& Kettner (2012).

Otro elemento importante para los funcionarios del PNNG son los mapas de probabilidad de inundación, los cuales se pueden convertir en una herramienta para la toma de decisiones en cuanto al traslado de nidos de tortugas. En este sentido, la zona media norte de la playa fue la que presentó menos riesgo de inundación y por tanto sería la zona más adecuada para 
conservar los nidos naturales y reubicar los que se encuentren en zonas de mayor riesgo. Algunos autores han estudiado el clima del oleaje cerca de la zona de estudio (e.g. Portilla, Sosaa \& Cavaleri, 2013) y la variabilidad de la cota de inundación (Agudelo, González \& Medina, 2004). Este estudio describió las condiciones de oleaje para isla Gorgona, estableciendo que en los trimestres DEF y MAM se presentó un oleaje con menor influencia de los vientos de la zona, en donde predominó el oleaje de fondo, mientras que en los trimestres JJA y SON se presentó una influencia directa del chorro de viento del Chocó.

Es importante destacar que para el desarrollo de esta investigación se consideraron varias supuestos. (1) la serie de oleaje se generó a partir de un modelo numérico que, aunque está basado en procesos físicos, fue alimentado con condiciones de frontera que provienen de reanálisis, es decir, que también son producto de modelación numérica. Sin embargo, los datos obtenidos del modelo fueron validados con información de campo. (2) Para el cálculo del run-up se usó una formulación empírica que, aunque describe bien el fenómeno, tiene algunas limitaciones, por ejemplo, solo considera la pendiente media y no la forma general del perfil de playa, y no considera la granulometría ni la permeabilidad del material, por lo que sobrestima los valores del run-up, ya que no se considera el volumen de agua que se infiltra. (3) Se consideró que el número de datos de la serie de cota de inundación es suficientemente largo para calcular la probabilidad como la frecuencia relativa y, por lo tanto, no se hizo un ajuste a una función de densidad conocida. (4) Los mapas de probabilidad de inundación, que son el producto final de este artículo, fueron creados con un levantamiento topográfico específico, pero debido a los cambios morfológicos de la playa, es necesario renovar estos mapas periódicamente teniendo en cuenta la escala temporal de variación de la morfología de la playa. (5) Al construir los mapas de probabilidad de inundación se trabajó bajo la suposición de que las características del oleaje no varían a lo largo de la playa, lo cual no es del todo cierto, ya que debido a procesos de transformación como difracción y asomeramiento, se presenta una variación de los parámetros del oleaje modificando espacialmente la cota de inundación.

La estimación de la cota de inundación es un problema importante y con aplicaciones variadas; en este caso se aplicó en la conservación de ecosistemas, pero se podría usar también como herramienta para la gestión del riesgo en general y el manejo integrado de zonas costeras. Por lo tanto hay que seguir investigando y aplicando nuevas metodologías, tanto en la obtención de los datos como en su tratamiento estadístico. Las líneas de investigación futuras apuntan hacia la implementación de modelos de inundación que, aplicados de manera sistemática, puedan predecir en tiempo real la inundación de una determinada playa, minimizando las parametrizaciones, simplificaciones y suposiciones.

\section{AGRADECIMIENTOS}

Al Programa Por Nuestros Mares, Fondo Alianza para la Acción Ambiental y la Niñez y Conservación Internacional por la financiación de este trabajo. Al Instituto de Hidráulica de la Universidad de Cantabria. De igual forma agradecen a todas las personas del PNN Gorgona y demás estudiantes del grupo OCEÁNICOS por sus valiosos aportes y decidido apoyo. Esta investigación estuvo amparada por el permiso de investigación de la UAESPNN No 008755 del 29 de septiembre de 2010. Al CWR de la Universidad de Australia Occidental por el uso del Modelo ELCOM.

\section{RESUMEN}

Playa Palmeras (En el Parque Nacional Isla Gorgona) es uno de los sitios más importantes para la anidación de tortugas marinas en América del Sur y Centroamérica. Debido a procesos morfológicos que afectan la playa, los nidos de las tortugas se han visto cada vez más expuestos al impacto del oleaje y la marea, poniendo en riesgo la conservación de éstas especies. Se llevó a cabo un estudio para conocer los procesos hidrodinámicos de la zona costera en Playa Palmeras, de los cuales depende el comportamiento 
morfodinámico de la playa y la preservación de los nidos. Se usó modelación numérica y mediciones en campo para conocer la variabilidad espacio-temporal del oleaje y obtener los patrones generales de circulación y la estructura termohalina de la zona. Se encontró un marcado ciclo anual, tanto en el oleaje como en las corrientes, influenciado por la Zona de Convergencia Intertropical (ZCIT) y una variabilidad interanual del oleaje, asociada a El Niño Oscilación del Sur (ENSO). Se estimó la cota de inundación de la playa y se crearon mapas de probabilidad de inundación, identificando los sitios potencialmente más seguros para la anidación. Los resultados muestran que hacia el norte de la playa está la zona de menor riesgo.

Palabras clave: modelización numérica, patrones de circulación, estructura termohalina, propagación de ondas, nivel de inundación, manejo de zonas costeras.

\section{REFERENCIAS}

Agudelo, P., González, M. \& Medina, R. (2004). Determinación de la cota de inundación del Pacífico Colombiano. XXI Congreso Latinoamericano de Hidráulica, São Pedro, Brasil.

Álvarez-Silva, O., Gómez-Giraldo, A. \& Toro, F. M. (2010). Calibración y Validación de un Modelo Hidrodinámico de la Desembocadura del Río León en el Golfo de Urabá, Colombia. XXIV Congreso Latinoamericano de Hidráulica, Punta del Este, Uruguay.

Amador, J. A., Alfaro, E. J., Lizano, O. G. \& Magaña V. O. (2006). Atmospheric forcing of the eastern tropical Pacific: A review. Progress in Oceanography, 69: 101-142.

Amante, C. \& Eakins, B. W. (2009). ETOPO1 1 Arc-Minute Global Relief Model: procedures, data sources and analysis. NOAA Technical Memorandum NESDIS NGDC-24.

Amorocho, D. F. \& Merizalde, L. A. (2004). Biología reproductiva de las tortugas marinas en el Parque Nacional Natural Gorgona: creando capacidad técnica para el manejo y la conservación de las tortugas marinas en hábitats reproductivos del Pacifico colombiano. Temporada Octubre-Noviembre, 2004. (Informe final). CIMAD-Conservación Internacional Colombia, Bogotá, Colombia.

Blanco, J. F. (2009). The hydroclimatology of Gorgona Island: seasonal and ENSO-related patterns. Actualidades Biológicas, 31: 111-121.

Booij, N., Ris, R. C. \& Holthuijsen, R. H. (1999). A third generation wave model for coastal regions, Part I, Model description and validation. Journal of Geophysical Research, 104: 7649-7666.

Casulli, V. \& Cheng, R. T. (1992). Semi-implicit finite difference methods for three-dimensional shallow water flow. International Journal for Numerical Methods in Fluids, 15: 629-648.

Chaigneau, A., Abarca del Rio, R. \& Colas, F. (2006). Lagrangian study of the Panama Bight and surrounding regions. Journal of Geophysical Research, 111: C09013

Correa I. D. \& Gonzalez, J. L. (2000). Coastal erosion and village relocation: a Colombian case study. Ocean \& Coastal Management, 43: 51-64.

Devis-Morales, A., Schneider, W., Montoya-Sánchez, R. A. \& Rodríguez-Rubio, E. (2008). Monsoon-like winds reverse oceanic circulation in the Panama Bight. Geophysical Research Letters, 35: L20607.

Giraldo, A., Rodríguez-Rubio, E. \& Zapata, F. (2008). Condiciones oceanográficas en isla Gorgona, Pacífico oriental tropical de Colombia. Latin American Journal of Aquatic Research, 36: 121-128.

Gómez-Giraldo, A., Osorio, A. F., Toro-Botero, F. M., Osorio, J. D., Álvarez-Silva, O. \& Arrieta, A. (2009). Patrón de circulación en Bahía Barbacoas y su influencia sobre el transporte de sedimentos hacia las islas del Rosario. Avances en Recursos Hidráulicos, 20: 21-39.

Giraldo, A., Payán, L. \& Chirimia, H. (2010). Variabilidad Térmica en el Ambiente Pelágico de Isla Gorgona entre marzo 2009-febrero 2010. In XIV Seminario Nacional de Ciencias y Tecnologías del Mar, Resúmenes Extendidos (pp. 75-80.). Cali, Colombia.

Hodges, B. \& Dallimore, C. (2006). Estuary, Lake and Coastal ocean model: ELCOM, science manual. Centre of Water Research: University of Western Australia.

Kalnay, E., Kanamitsu, M., Kistler, R., Collins, W., Deaven, D., Gandin, L., Iredell, M., Saha, S., White, G., Woollen, J., Zhu, Y., Leetmaa, A., Reynolds, R., Chelliah, M., Ebisuzaki, W., Higgins, W., Janowiak, J., Mo, K. C., Ropelewski, C., Wang, J., Jenne, R. \& Joseph, D. (1996). The NCEP/NCAR 40-year reanalysis project. Bulletin of the American Meteorological Society, 77: 437-471.

Kessler, W. S. (2006). The circulation of the eastern tropical Pacific: A review. Progress in Oceanography, 69: 181-217.

Le Provost, C. (2001). Ocean tides. In: Satellite altimetry and earth sciences, a handbook of techniques and applications. Geophysical Journal International, 69: 267-303.

Martínez J. O., González, J., Pilkey, O. H. \& Neal, W. J. (1995). Tropical barrier islands of Colombia's Pacific Coast. Journal of Coastal Research, 11 (2): 432-453.

Martínez J. O. \& López-Ramos, E. (2011). High-resolution seismic stratigraphy of the late Neogene of the central sector of the Colombian Pacific continental shelf: A seismic expression of an active continental 
margin. Journal of South American Earth Sciences, 31: 28-44.

Nielsen, P. \& D. J. Hanslow. (1991). Wave run-up distributions on natural beaches. Journal of Coastal Research, 7 (4):1139-1152.

Montoya, L. J. \& Toro, F. M. (2006). Calibración de un modelo hidrodinámico para el estudio de los patrones de circulación en el Golfo de Urabá. Revista Avances en Recursos Hidráulicos, 13: 37-54.

Portilla J., Sosaa J. \& Cavaleri, L. (2013). Wave energy resources: Wave climate and exploitation. Renewable Energy, 57: 594-605.

Poveda, G \& Mesa, O. J. (1999). La corriente del Oeste ("Chocó") y otras dos corrientes de chorro en Colombia: Climatología y variabilidad durante las fases del ENSO. Revista de la Academia Colombiana de Ciencias Exactas, Físicas y Naturales, 23(89): 517-529

Poveda, G. (2004). La Hidroclimatología de Colombia: una sintesis desde la escala inter-decadal hasta la escala diurna. Revista de la Academia Colombiana de Ciencias Exactas, Físicas y Naturales, 28 (107): 201-222.

Restrepo, J. D., \& Kettner, A. (2012). Human induced discharge diversion in a tropical delta and its environmental implications: The Patía River, Colombia. Journal of Hydrology, 424:124-142.

Rodríguez-Rubio, E. (2013). A multivariate climate index for the western coast of Colombia. Advances in Geosciences, 33(33):21-26.

Rodríguez-Rubio, E. \& Stuardo, J. (2002). Variability of photosynthetic pigments in the Colombian Pacific
Ocean and its relationship with the wind field using ADEOS-I data, Proceedings of the Indian Academy of Science, 111(3): 227-236.

Rodríguez-Rubio, E., Schneider, W. \& Abarca del Rio, R. (2003). On the seasonal circulation within the Panama Bight derived from satellite observations of wind, altimetry and sea surface temperature. Geophysical Research Letters, 30(7): 1410.

Reguero, B. G., Menéndez, M., Méndez, F. J., Mínguez, R. \& Losada, I. J. (2012). A Global Ocean Wave (GOW) calibrated reanalysis from 1948 onwards. Coastal Engineering, 65: 38-55.

Restrepo J. D. \& Kettner A. (2012). Human induced discharge diversion in a tropical delta and its environmental implications: The Patía River, Colombia. Journal of Hydrology, 424-425: 124-142.

Rueda-Almonacid, J. V., Carr, J. L., Mittermeier, R. A., Rodríguez-Mahecha, J. V., Mast, R. B., Vogt, R. C., Rhodin, A. G. J., de la Ossa-Velásquez, J., Rueda, J. N. \& Mittermeier, C. G. (2007). Las tortugas y los cocodrilianos de los países andinos del trópico. Conservación Internacional, Bogotá, Colombia.

The SWAN Team. (2009). SWAN Technical Documentation. Delft University of Technology. Delft, Holland.

Wolter, K. \& Timlin, M. S. (1998). Measuring the strength of ENSO - how does 1997/98 rank?. Weather, 53: 315-324

Wyrtki, K. (1966). Oceanography of the eastern equatorial Pacific Ocean. Journal of Marine Biology \& Oceanography, 4: 33-68. 
